\title{
TRES INSCRIPCiONES ROMANAS DE LA PROVINCIA DE CuenCA
}

\author{
JUAN MANUEL ABASCAL PALAZÓN¹, MIGUEL ÁNGEL VALERO TÉVAR²
}

(1) Área de Historia Antigua. Universidad de Alicante.juan.abascal@ua.es

(2) Área de Historia Antigua. Universidad de Castilla-La Mancha. MiguelAngel.Valero@uclm.es

La provincia de Cuenca reúne en estos momentos algo más de 1200 inscripciones romanas, de las que la mayor parte procede de Segobriga y, en menor medida, de Valeria. Eso puede dar idea del elevado hábito epigráfico de la región y explica la sucesión de hallazgos ocasionales de nuevos testimonios como los que presentamos ${ }^{1}$.

\section{LEDAÑA (fig. 1)}

Estela de piedra caliza y cabecera triangular, partida en dos fragmentos que encajan entre sí. Está alisada por los lados estrechos, pero la parte posterior tiene un tratamiento menos cuidadoso. Sus dimensiones son 61 x 53 x $28 \mathrm{~cm}$. La altura de las letras es de $5 \mathrm{~cm}$ en el primer renglón, 4 en el segundo y ca. 3,5/4 en el tercero. Las interpunciones son circulares. Por debajo del último renglón hay un espacio libre de unos $32 \mathrm{~cm}$ de altura.

Fue descubierta en Ledaña, en los años 90 del siglo pasado, en las obras de cimentación de una vivienda, situada en la esquina de las calles Manuel Attard y Serrano Morales (coordenadas: 39²1'25” N, 142' 56” W).

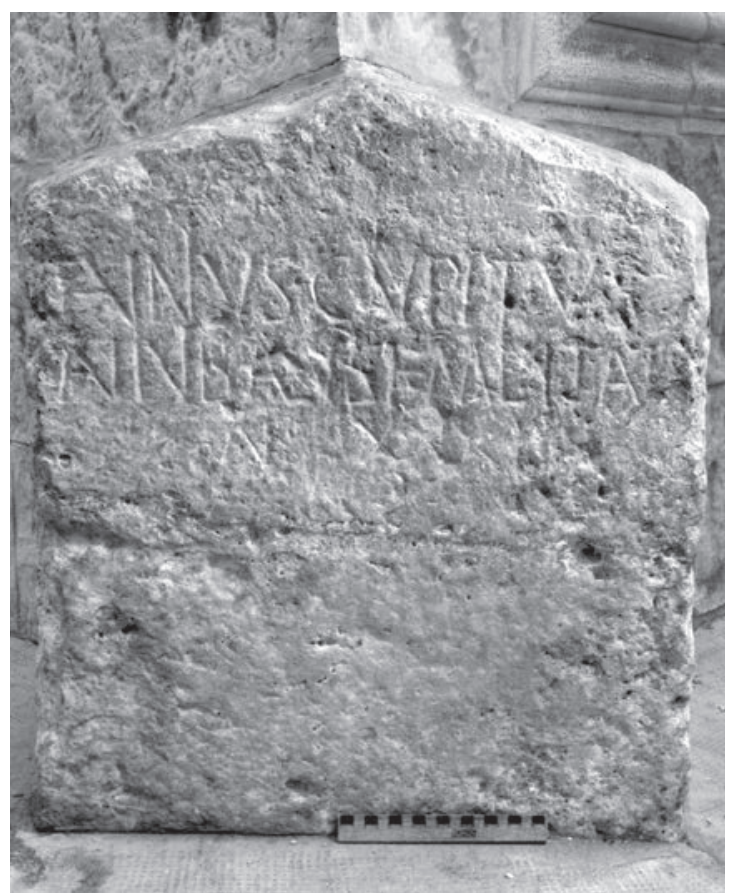

Fig. 1: Estela de Ledaña (Foto: J. M. Abascal).

Copyright: (c) 2017 Juan Manuel Abascal \& Miguel Ángel Valero. This is an open access paper distributed under the terms of the Creative Commons License, (CC BY-NC-SA 3.0), which permits unrestricted use, distribution, and reproduction in any medium, provided the original author and source are credited. 


\section{Ânnius $\cdot$ Cupitus \\ Annibâlis $\cdot$ f(ilius) militâv(it) \\ an(nis) XXXI}

Se conserva allí mismo, encastrada en el ángulo exterior de la casa, en donde la describimos y fotografiamos el 4 de junio de 2017.

1 Nexos AN y NI. - 2 Nexos AN, NI, AL, IL, AV. - 3 Nexo AN.

El cognomen Hannibal no es desconocido pues, entre otros textos, se repite en una inscripción de Roma (CIL VI 6461), en AE 1979, 271 de Oderzo (Italia, regio X), etc.

Dado que Annius Cupitus carece de praenomen, con toda probabilidad fue soldado de una cohorte o ala auxiliar y falleció antes de su licenciamiento; la ausencia de fórmula funeraria parece indicar que estamos ante un cenotafio.

El tipo de letra aconseja datar la estela en el s. II.

A unos $1300 \mathrm{~m}$ al $\mathrm{N}$ del lugar de hallazgo de esta estela se encuentra el yacimiento de Los Villares, con unas dimensiones de casi 24 ha y numerosos restos cerámicos en superficie. El lugar ha sido prospectado en el ámbito de proyectos como "El territorio ibérico en la Manchuela"2 (Valero 2008: 160 ss.) o "Carta Arqueológica de Ledaña"3, lo que ha permitido el análisis de una muestra de material cerámico que, pese a ser aleatoria, indica una presencia humana en tiempos ibéricos y una mayor intensidad de ocupación en época romana. No existen otros testimonios que apunten a la presencia de un yacimiento bajo el trazado urbano de la localidad. En todo caso, el hallazgo de la estela de un soldado sin ciudadanía, es decir, habitante de un enclave estipendiario, apunta a la existencia aquí de una necrópolis asociada al enclave de Los Villares ya citado.

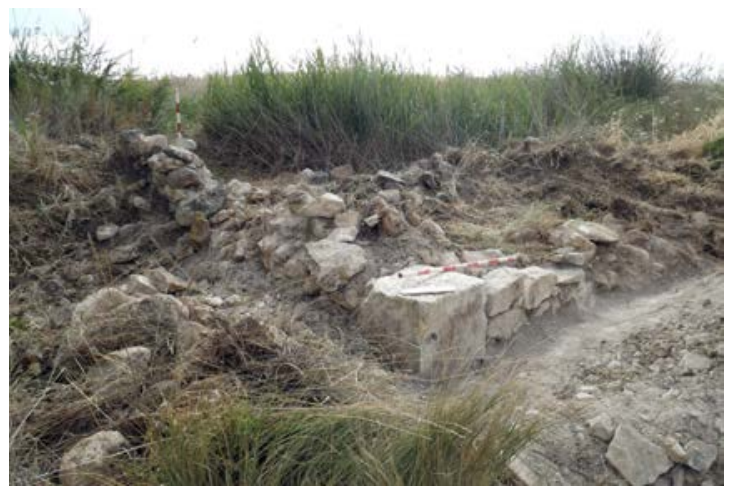

Fig. 2: Altar de Villarejo de Fuentes in situ en el momento del descubrimiento (Foto: M. Á. Valero).
Hoy por hoy desconocemos el nombre del municipium al que pudieron pertenecer tanto estas tierras de Ledaña como las cercanas de Iniesta (Valero 1995; 2010) y de muchos enclaves de La Manchuela en los que hay evidencias epigráficas.

\section{VILLAREJO DE FUENTES (figs. 2-4)}

Altar de caliza retallada para convertirlo en un bloque regular de construcción, de manera que ha perdido el coronamiento y el zócalo ha sido reducido para adaptarlo a las dimensiones del fuste. No obstante, las huellas de talla muestran que antiguamente las molduras del zócalo no sobresalían por la parte posterior, de lo que hay que deducir que el monumento estuvo originalmente adosado a una pared. En el costado izquierdo del fuste presenta una fractura que no afecta al texto, pero en su parte derecha los golpes han hecho desaparecer la $\mathrm{S}$ final del nomen gentile del primer dedicante y han dañado la letra $\mathrm{T}$ de la tercera línea conservada. Sus dimensiones actuales son (63) x (52) x $43 \mathrm{~cm}$ y la altura de las letras es de $4,5 \mathrm{~cm}$ en todas las líneas. Presenta interpunciones circulares.

El epígrafe apareció en los trabajos de control arqueológico de las obras de acondicionamiento de la carretera CM-3118 durante el año 2014 ${ }^{4}$. El bloque con la inscripción estaba reutilizado en el ángulo del zócalo en una pequeña edificación agrícola ya destruida, situada cerca de una surgencia de agua denominada Fuente de la Pioja, dentro del término municipal de Villarejo de Fuentes (coordenadas: $39^{\circ} 45^{\prime} 27^{\prime \prime} \mathrm{N}, 2^{\circ} 37^{\prime} 08^{\prime \prime} \mathrm{W}$ ) (fig. 2). Lo describimos y fotografiamos el 12 de junio de 2017. Se encuentra ya depositado en el Museo de Cuenca.

Los cognomina griegos Dorus (Solin 2003: 512) y Pistus (Ibid.: 793) ya eran conocidos con anterioridad en inscripciones de Hispania (Abascal 1994: 347 y 459 respectivamente).

La forma de las letras aconseja datar este monumento a mediados o en la segunda mitad del s. I.

Hasta el momento no se tenía constancia del hallazgo de inscripciones en la localidad del Villarejo de Fuentes, excepción hecha de la falsa noticia sobre el descubrimiento de un miliario en el s. XVII (CIL II 465*; Curchin 1985: 246, n. ${ }^{\circ}$ 10a; Lostal 1992: 223 n. ${ }^{\circ} 272$ ), aunque sí en el vecino Villar de Cañas (Osuna y Suay 1974: s/p; Abascal 2013: 24-25, n. $\left.{ }^{\circ} \mathrm{VI}\right)$; ambos municipios se encuentran dentro del territorium de Segobriga. 


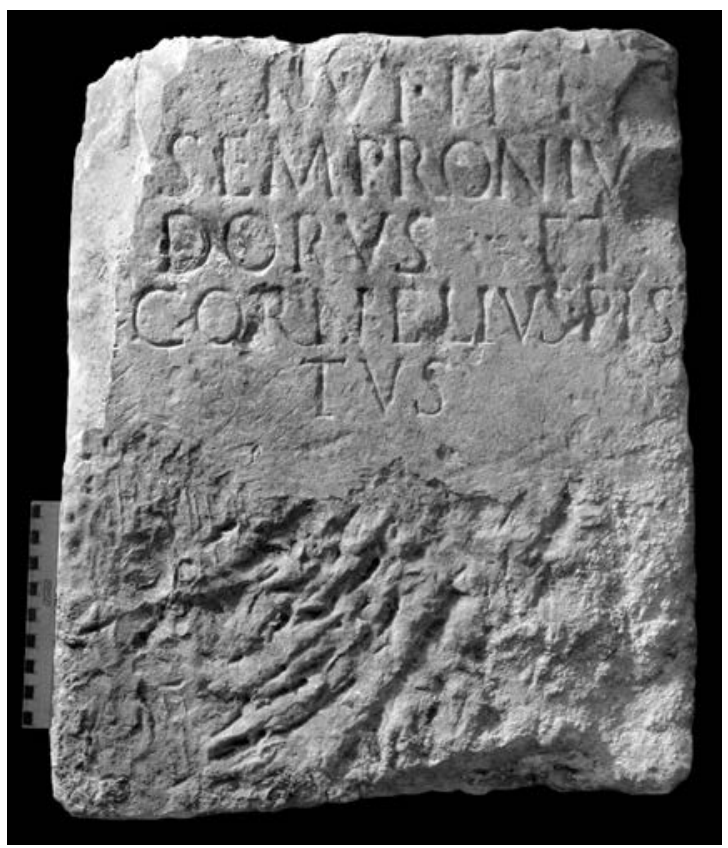

Fig. 3: Monumento funerario de Villarejo de Fuentes (Foto: J. M. Abascal).

$$
\begin{array}{ll} 
& {[-----]} \\
& {[---f e c e]-} \\
\text { runt } & \\
& \text { Semproniu[s] } \\
& \text { Donus } \cdot \text { et } \\
& \text { Cornelius } \cdot \text { Pis- } \\
5 & \text { tus }
\end{array}
$$

Tal y como se ha apuntado, el epígrafe fue reutilizado, y por tanto no se encontraba en su lugar de origen, pero a unos $200 \mathrm{~m}$ al $\mathrm{S}$ de este punto se localiza el yacimiento de La Pioja, en el mismo término municipal de Villarejo de Fuentes. Dicho enclave se sitúa en la parte alta y media de un promontorio y el lugar ha sido estudiado en dos ocasiones; la primera, con motivo de la realización de la Carta Arqueológica de Villarejo de Fuentes ${ }^{5}$, y la segunda con ocasión de los citados trabajos preventivos inherentes a las obras públicas, lo que permite poder adscribirlo a época romana (ss. I-III), contando con un área de dispersión cerámica de unas 2 ha. Por otro lado, a unos $800 \mathrm{~m}$ al $\mathrm{O}$ del mismo se encuentra otro enclave de la misma cronología, pero con una mayor presencia de material cerámico, entre el que desatacan los fragmentos de terra sigillata hispánica, ánforas, dolia, cerámica común, etc., a lo que hay que sumar una ingente cantidad de restos de tegulae.

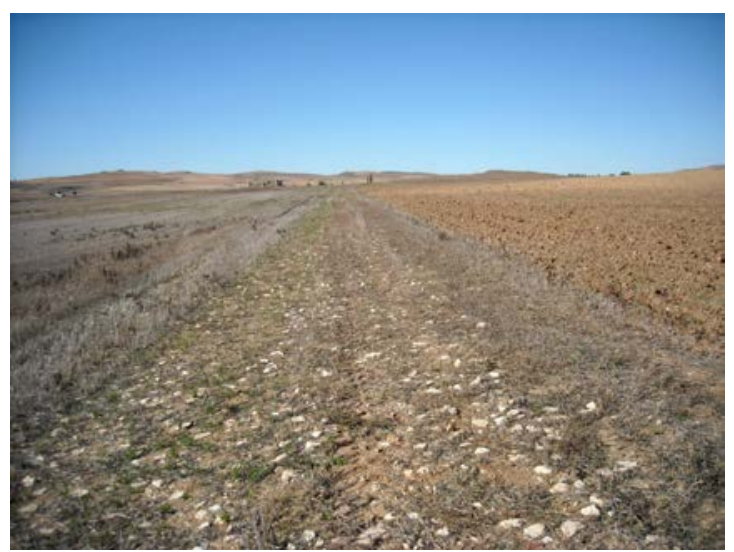

Fig. 4: El llamado "Camino romano" en término de Villarejo de Fuentes (Foto: M. Á. Valero).

La superficie del mismo es de unas 28 ha. A todo ello se suma que entre ambos se ubica el denominado "Camino romano" (Palomero 1987: 112), la vía histórica que se dirige a Segobriga y que en la actualidad se encuentra en desuso (fig. 4). En cualquier caso, hay que resaltar la densa ocupación histórica de este espacio y la posibilidad de que existiera aquí algún tipo de población romana.

\section{CARBONERAS DE GUADAZAÓN (fig. 5)}

Bloque de piedra caliza blanquecina, poco alisado por los lados y por la parte posterior, con numerosos daños en la parte frontal y en el extremo superior, que han hecho desaparecer algunas letras. Por arriba conserva vestigios de opus caementicium antiguo, por lo que debía formar parte de un monumento de mayores dimensiones y compuesto de varios bloques. Sus dimensiones actuales son $75 \times 52 \times 26 \mathrm{~cm}$. El espacio destinado a la escritura está ligeramente rebajado y tiene forma de tabula ansata; mide (46) x 38. La altura de las letras es de $5,5 \mathrm{~cm}$ en los dos primeros renglones y de $5 \mathrm{~cm}$ en los dos siguientes; las interpunciones fueron circulares, aunque hoy parecen deformes.

El epígrafe apareció de manera fortuita al realizar labores agrícolas en el paraje de Los Prados, cerca del yacimiento arqueológico de Villar de Cañas III (coordenadas: $39^{\circ} 55^{\prime} 15^{\prime} \mathrm{N}, 1^{\circ} 49^{\prime} 34^{\prime}$ ' W), que había sido localizado al realizar la Carta Arqueológica de Carboneras de Guadazaón ${ }^{6}$; los hallazgos en superficie aseguran su datación en época romana y una extensión de $c a .1,44$ ha. 


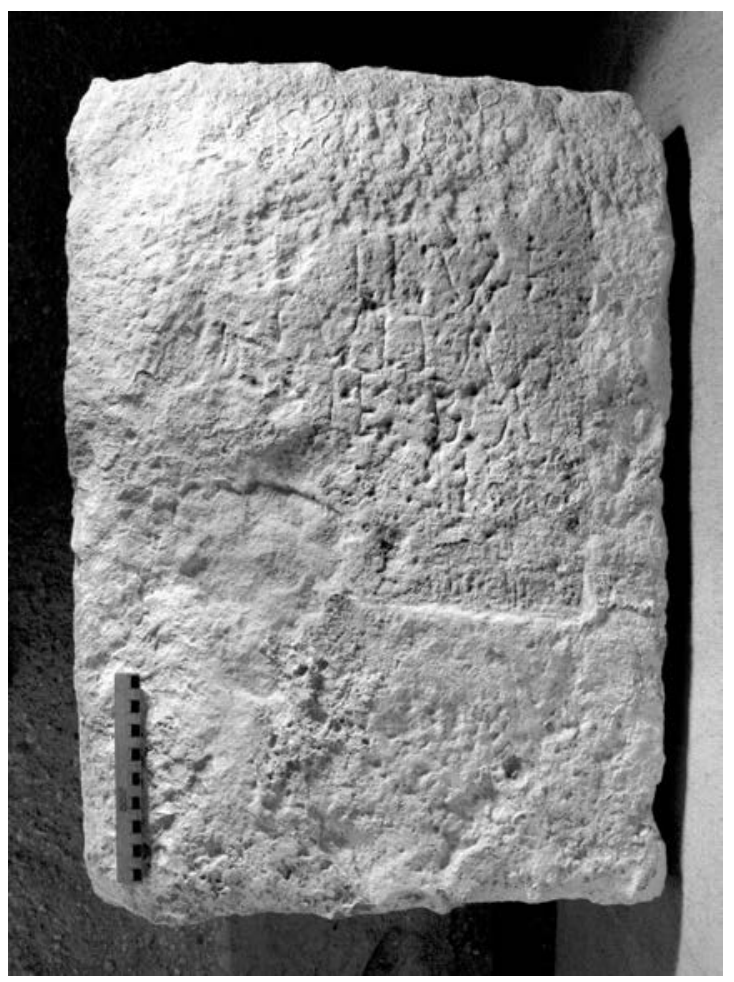

Fig. 5: Estela funeraria de Carboneras (Foto: J. M. Abascal).

$$
\begin{aligned}
& -----? \\
& {[---] L L V \cdot f(\text { ili- })} \\
& {[p o] s ̦ u i t \cdot m a ̣-} \\
& {[t--]+E \cdot B A[-]} \\
& {[---] \text { h(ic) }[s(\text { it- }) e(s t)]} \\
& -----?
\end{aligned}
$$

El lugar perteneció en la antigüedad al territorium de la ciudad de Valeria. El epígrafe se conserva en Carboneras en propiedad particular; allí lo pudimos describir y fotografiar el 12 de junio de 2017.

En el primer renglón debía encontrarse el nombre del difunto escrito irregularmente con un dativo en $-u$. Es menos probable que se trate de un dedicante masculino con filiación. En la tercera línea la cruz identifica el resto de una probable $S$.

El tipo de monumento y la forma de las letras aconsejan datar la estela a mediados o en la segunda mitad del s. II.

Hasta el momento conocíamos en esta localidad otras cuatro inscripciones: la estela de Fortilla (AE 1982: 608) y el monumento funerario de Perennis (AE 1982: 609) proceden de lugares imprecisos del término municipal; en el s. XVIII apareció la estela de Terentia junto a la ermita de San Bartolomé (CIL II 3007) y en el mismo enclave que ahora nos ocupa, el paraje de Villar de Cañas, se encontró también en el s. XVIII el altar funerario hexagonal dedicado por Secundina (AE 1982: 610). Se tiene constancia de la aparición a lo largo de los años de otras estelas hoy perdidas en este yacimiento. Pero en este análisis también se ha de considerar la presencia -a escasos $500 \mathrm{~m}$ al $\mathrm{O}$ del punto de aparición del monumento epigráfico- de un yacimiento de 24 ha de dispersión de hallazgos cerámicos, con evidencias de ocupación desde época ibérica hasta mediados del s. III d.C.

\section{NOTAS}

1. Este trabajo se ha realizado en el marco del proyecto de investigación Sociedad romana y hábito epigráfico en la Hispania citerior, HAR2015-65168-P (MINECO/FEDER), subvencionado por el Ministerio de Economía y Competitividad del Gobierno de España.

2. Este proyecto fue dirigido por Miguel Ángel Valero Tévar, desarrollándose entre los años 1996-1998.

3. La Carta Arqueológica fue elaborada por un amplio equipo de profesionales que contó con la dirección de Miguel Ángel Valero Tévar. Dicho trabajo se efectuó por encargo del Ayuntamiento de Ledaña entre los años 2007 y 2008, siendo financiado por el Grupo de Acción Local ADIMAN.

4. La aparición del epígrafe de adscripción romana motivó la paralización cautelar de los trabajos en la zona del descubrimiento, notificándose al Servicio de Patrimonio de la Dirección Provincial en Cuenca de la Consejería de Educación, Cultura y Deporte de Castilla-La Mancha.

5. La Carta Arqueológica de Villarejo de Fuentes fue elaborada por un amplio equipo de profesionales bajo la dirección de Miguel Ángel Valero Tévar. Dicho trabajo se efectuó por encargo del Ayuntamiento de la localidad entre los años 2010 y 2011, siendo financiado por el Grupo de Acción Local ADIMMAC.

6. La Carta Arqueológica de Carboneras de Guadazaón, como en los casos anteriores fue elaborada por un amplio equipo de profesionales bajo la dirección de Miguel Ángel Valero Tévar. Dicho trabajo se efectuó por encargo del Ayuntamiento de la localidad entre los años 2007 y 2008, siendo financiado por el Grupo de Acción Local PRODESE.

\section{BIBLIOGRAFÍA}

ABASCAL, J. M. (1994): Los nombres personales en las inscripciones latinas de Hispania, Murcia.

ABASCAL, J. M. (2013): Cuestiones epigráficas del conventus Carthaginiensis (Hispania citerior), con algunas contribuciones póstumas de Géza Alföldy, Actes Ier Congrés Internacional d'Arqueologia i món antic. Govern i societat a la Hispània romana. Novetats epigràfiques. Homenatge a Géza Alföldy (J. López Vilar, ed.), Tarragona, 13-34. 
CURCHIN, L. A. (1985): Epigraphic notes from the Spanish Meseta, ZPE 58, 244-246.

LOSTAL, J. (1992), Los miliarios de la provincia Tarraconense (Conventos Tarraconense, Caesaraugustano, Cluniense y Cartaginense), Zaragoza.

OSUNA, M.; SUAY, F. (1974): Yacimientos romanos de la provincia de Cuenca, Cuenca 6, 25-59.

PALOMERO, S. (1987): Las vías romanas de la provincia de Cuenca, Cuenca.

SOLIN, H. (2003): Die griechischen Personennamen in Rom. Zweite, völlig neu bearbeitete Auflage I-III, Berlin-New York.
VALERO, M. Á. (1995): Una inscripción funeraria procedente de Iniesta (Cuenca), Saguntum-PLAV 25, 283-286.

VALERO, M. Á. (2008): El territorio ibérico en Las Manchuela: avance de los primeros resultados, Studia Academica 1, $155-195$.

VALERO, M. Á. (2010): La necrópolis ibérica de La Punta del Barrionuevo, Iniesta-Cuenca: Avance de las últimas investigaciones, Actas de las II Jornadas de Arqueología de Castilla-La Mancha, Toledo, 1010-1045. 